TAUP 2186-94

\title{
Charges, Monopoles and Duality Relations
}

\author{
E. Comay \\ School of Physics and Astronomy \\ Raymond and Beverly Sackler Faculty \\ of Exact Sciences \\ Tel Aviv University \\ Tel Aviv 69978 \\ Israel
}

PACS No: 03.30.+p, 03.50.De, 14.80.Hv

\begin{abstract}
:
A charge-monopole theory is derived from simple and self-evident postulates. Charges and monopoles take an analogous theoretical structure. It is proved that charges interact with free waves emitted from monopoles but not with the corresponding velocity fields. Analogous relations hold for monopole equations of motion. The system's equations of motion can be derived from a regular Lagrangian function.
\end{abstract}




\section{Introduction}

The problem of a theoretical formulation of the behavior of a chargemonopole system is known for a long time. Many discussions on the subject can be found in the literature[1,2]. One unsettled point of many chargemonopole theories is the lack of a regular classical Lagrangian function from which the theory can be derived $[3,4]$. This shortcoming entails difficulties in a construction of a quantum mechanical theory of the system $[5,6]$.

An attempt to overcome this problem has been carried out several years ago[4]. This approach relies on the experimental fact which says that monopoles have not been seen in laboratories. Hence, their equations of motion have not been confirmed in experiment. It follows that one is not obliged to construct the theory from a specific form of the equations of motion. Thus, the approach of [4] is based on the following postulates:

A. A system of monopoles and fields is dual to a system of charges and fields.

B. The equations of motion of a system of charges, monopoles and fields can be derived from a regular Lagrangian function.

In addition to these postulates, several other self-evident ones are used. It is shown in [4] that one can construct a self-consistent theory on this basis. As postulated, this theory is derived from a regular Lagrangian function.

The purpose of the present work is to investigate the possibility of constructing the same theory without relying on postulate B. Although the assumption of the existence of a regular Lagrangian function is appealing in its own, it is clear that the results are stronger if they are obtained on the basis of fewer postulates. In particular, the existence of a regular Lagrangian 
function is deduced from the set of postulates used here.

In the present work, expressions are written in units where the speed of light $c$ equals unity. Greek indices range from 0 to 3 . The metric is diagonal and its entries are $(1,-1,-1,-1)$. The symbol,$\nu$ denotes the partial differentiation with respect to $x^{\nu}$. $\tau$ denotes the invariant time.

The paper is organized as follows. Theories of two different kinds of charges are presented in the second sections. A theory of a combined system of charges and monopoles is derived in the third section. Consequences of the results are discussed in the fourth section. The last section contains concluding remarks.

\section{Two Kinds of Charges}

Consider a system of one kind of charges and of their associated fields which satisfy the equations of motion of classical electrodynamics. Here charges are the well known electric charges. The Lorentz force exerted on charges is

$$
\frac{d P_{(e)}^{\mu}}{d \tau}=F_{(e)}^{\mu \nu} J_{(e) \nu} .
$$

The subscript $(e)$ denotes that the quantities belong to a system of charges. The entries of the fields tensor of (1) are

$$
F_{(e)}^{\mu \nu}=\left(\begin{array}{cccc}
0 & -E_{x} & -E_{y} & -E_{z} \\
E_{x} & 0 & -B_{z} & B_{y} \\
E_{y} & B_{z} & 0 & -B_{x} \\
E_{z} & -B_{y} & B_{x} & 0
\end{array}\right)
$$

As is well known, physics is a science which relies on experiment. Hence, physical quantities should be related to measurements. The Lorentz force (1) can be used for measuring the fields. This objective can be achieved by means 
of a set of measurements that yield unique values of the six independent entries of the antisymmetric fields tensor $F_{(e)}^{\mu \nu}$. Thus, the fields are defined by means of (11) [7].

The fields satisfy Maxwell equations

$$
\begin{gathered}
F_{(e), \nu}^{\mu \nu}=-4 \pi J_{(e)}^{\mu} \\
F_{(e), \nu}^{* \mu \nu}=0 .
\end{gathered}
$$

Here

$$
F_{(e)}^{* \mu \nu}=\frac{1}{2} \varepsilon^{\mu \nu \alpha \beta} F_{(e) \alpha \beta}=\left(\begin{array}{cccc}
0 & -B_{x} & -B_{y} & -B_{z} \\
B_{x} & 0 & E_{z} & -E_{y} \\
B_{y} & -E_{z} & 0 & E_{x} \\
B_{z} & E_{y} & -E_{x} & 0
\end{array}\right)
$$

and $\varepsilon^{\mu \nu \alpha \beta}$ is the completely antisymmetric unit tensor of the fourth rank. The fields can be derived from a regular 4-potential[8] (Point singularities associated with an elementary classical point charge are not discussed in the present work)

$$
F_{(e) \mu \nu}=A_{(e) \nu, \mu}-A_{(e) \mu, \nu} .
$$

An important quantity is the fields energy-momentum tensor. This quantity represents the density and the current of energy and momentum throughout space-time. Its form is (see [7], p.605 or [8], p.81)

$$
T_{(e)}^{\mu \nu}=\frac{1}{4 \pi}\left(F_{(e)}^{\mu \alpha} F_{(e)}^{\beta \nu} g_{\alpha \beta}+\frac{1}{4} F_{(e)}^{\alpha \beta} F_{(e) \alpha \beta} g^{\mu \nu}\right)
$$

Let us consider another system of charges whose type is not known yet. The second system is isolated from the electromagnetic system described above and is denoted by the subscript $(m)$. Interactions within the $(m)$ system are formally the same as those of the electromagnetic system $(e)$. Thus, the foregoing laws hold for the new system and the sole change is the replacement of the subscript $(e)$ by $(m)$. 
Like the fields of the electromagnetic system $(e)$, the fields of the $(m)$ system are defined by the force exerted on a charge. This quantity is written in an ordinary tensorial form which is analogous to (四)

$$
\frac{d P_{(m)}^{\mu}}{d \tau}=F_{(m)}^{\mu \nu} J_{(m) \nu}
$$

The fields equations of motion take the form of Maxwell equations

$$
\begin{gathered}
F_{(m), \nu}^{\mu \nu}=-4 \pi J_{(m)}^{\mu} \\
F_{(m), \nu}^{* \mu \nu}=0 .
\end{gathered}
$$

These fields can be derived from a regular potential which is a 4-vector

$$
F_{(m) \mu \nu}=A_{(m) \nu, \mu}-A_{(m) \mu, \nu}
$$

Other quantities of the $(m)$ system, like the energy-momentum tensor, are also analogous to the corresponding ones of the $(e)$ system.

So far nothing has been said on the interrelations between the $(e)$ system and the $(m)$ one. Three possible relations are as follows.

- Charges of the $(m)$ system are electric charges.

- Charges and fields of the $(m)$ system do not interact with the electromagnetic system $(e)$.

- Charges of the $(m)$ system are magnetic monopoles.

In the first case, there is nothing to add, because problems of electrodynamics of a pure system of charges is outside the scope of the present work. In the second case, the $(m)$ system cannot be seen in electromagnetic processes and it behaves as a dark matter. The rest of this work is devoted to 
interrelations between electric charges and magnetic monopoles.

\section{Interactions of a Charge-Monopole System}

Consider a system $\{e\}$ of charges without monopoles and a system $\{m\}$ of monopoles without charges. One can imagine that these systems are very far from each other. At the limit of infinite distance the two systems do not interact and the theory of the combined system is just the two separate theories described in the previous section. As $\{e\}$ and $\{m\}$ move towards each other the interaction between these systems increases. Thus, the theory of the combined system contains the theories of $\{e\}$ and of $\{m\}$ as two subtheories obtained in the limit where the two subsystems are infinitely far apart.

It can be shown[1] that duality transformations cast electrodynamics of charges into electrodynamics of monopoles. Thus, in the notation used here, these transformations take the form

$$
\begin{gathered}
\boldsymbol{E}_{(e)} \rightarrow \boldsymbol{B}_{(m)}, \\
\boldsymbol{B}_{(e)} \rightarrow-\boldsymbol{E}_{(m)},
\end{gathered}
$$

and

$$
e \rightarrow g
$$

where $g$ denotes the strength of magnetic charges. In the previous section, notation of the entries of the fields tensor of the $\{m\}$ system is not specified. Thus, its entries are denoted here like those of the dual tensor (5) of the $\{e\}$ 
system

$$
F_{(m)}^{\mu \nu}=\left(\begin{array}{cccc}
0 & -B_{x} & -B_{y} & -B_{z} \\
B_{x} & 0 & E_{z} & -E_{y} \\
B_{y} & -E_{z} & 0 & E_{x} \\
B_{z} & E_{y} & -E_{x} & 0
\end{array}\right) .
$$

In this way the notation of charges' and monopoles' fields, which are defined by (11) and (8), respectively, take a related form as seen in the ordinary tensors (2) and (15), respectively.

In the rest of this section, it is found how the $\{e\}$ and $\{m\}$ systems can interact without destroying their intrinsic properties as formulated in the previous section. To this end, the problem is divided into two parts. The linearity of classical electrodynamics enables the split of the fields into two subsets. One of these subsets is made of the velocity fields of charges and the other subset is made of the acceleration fields of charges. An analogous division takes place in the case of monopoles' fields.

Velocity fields and acceleration fields are seen in the Lienard-Wiechert expression for retarded field of a charge $q$ (see [7], p. 657 or [8], p.162)

$$
\begin{gathered}
\boldsymbol{E}=q\left[\frac{1-v^{2}}{(R-\boldsymbol{R} \cdot \boldsymbol{v})^{3}}(\boldsymbol{R}-R \boldsymbol{v})+\frac{1}{(R-\boldsymbol{R} \cdot \boldsymbol{v})^{3}} \boldsymbol{R} \times\{(\boldsymbol{R}-R \boldsymbol{v}) \times \boldsymbol{a}\}\right] \\
\boldsymbol{B}=\boldsymbol{R} \times \boldsymbol{E} / R .
\end{gathered}
$$

Here $\boldsymbol{R}$ denotes the radius-vector from the retarded position of the charge $q$ to the point where the fields are calculated and $\boldsymbol{v}$ and $\boldsymbol{a}$ denote the retarded velocity and acceleration of $q$, respectively. Analogous expressions hold for fields of monopoles.

The first term on the right hand side of (16) and of (17) is called a velocity field and the corresponding second term is called acceleration field (see [7], p.657). The acceleration fields decrease like $R^{-1}$. Hence, the energy density of these fields $T^{00}=\left(E^{2}+B^{2}\right) / 8 \pi$ decreases like $R^{-2}$. The same property holds for all entries of the energy-momentum tensor (7) of acceleration fields. It 
follows that these fields represent an objective physical entity that is endowed with an outgoing flux of energy and momentum. On the other hand, energy density of velocity fields as well as the interaction energy density of velocity fields and acceleration fields decrease like $R^{-4}$ and $R^{-3}$, respectively. It follows that the energy flux of these fields vanishes at infinity. This discussion shows that acceleration fields are dominant at very far regions and represent free electromagnetic waves. In quantum mechanics they take the form of real photons. The portion of space which is very far from the origin is the wave zone. Henceforth, acceleration fields at the wave zone are called free waves.

Let us examine free waves. Consider a monochromatic plane electromagnetic wave emitted from a very far charge (see [8] pp. 114,115). The wave moves in the z-direction and its fields are

$$
\begin{aligned}
& \boldsymbol{E}=(1,0,0) \sin [\omega(t-z)] \\
& \boldsymbol{B}=(0,1,0) \sin [\omega(t-z)] .
\end{aligned}
$$

Relation (6) shows that these fields, which are written here in a 3 -vector notation, can be derived from the following 4-potential

$$
A_{(e) \mu}=(0,1,0,0) \cos [\omega(t-z)] / \omega
$$

As seen from the components of $\boldsymbol{E}$ and of the 4-potential (20), this field is polarized in the x-direction.

Let us examine how the free wave whose fields are (18) and (19) is seen in the $\{m\}$ system. For this purpose consider the 4-potential

$$
A_{(m) \mu}=(0,0,1,0) \cos [\omega(t-z)] / \omega .
$$

An application of (11) and of the notation (15) of the entries of the monopole fields tensor, shows that the electric and the magnetic fields (18) and (19) 
are obtained from the 4-potential (21). On the basis of these results it is concluded here that free waves of charges look the same as free waves of monopoles.

Let us turn to velocity fields of the systems. The problem of interaction of monopoles with velocity fields of charges is analyzed by means of the following example. Consider two magnetic polar dipoles made of monopole matter. One face of each dipole is covered with $+g$ magnetic monopoles and the opposite face is covered with $-g$ magnetic monopoles. Let $p$ denote the strength of the dipoles. In a coordinate frame $\Sigma$, the dipoles are placed as depicted in Fig. 1.a. The force exerted on the upper dipole is the same as the force found in an analogous system of polar electric dipoles[9]. Using also the well known expression for dipole field (see [7], p. 141), one finds the required force

$$
\boldsymbol{f}=(0,-1,0) 3 p^{2} /\left(8 r^{4}\right)
$$

The force exerted on the lower dipole has the same size and takes the opposite direction. It follows that the two dipoles attract each other.

Let us examine the same system in another coordinate frame $\Sigma^{\prime}$. This frame is related to $\Sigma$ by means of space reflection. Thus, $\Sigma$ is a right handed frame and $\Sigma^{\prime}$ is a left handed frame. Obviously, the frame $\Sigma^{\prime}$, like $\Sigma$, is a legitimate frame and the laws of physics should hold in it.

On the basis of the tensorial relations (8)-(11) it is found that, in $\Sigma^{\prime}$, the dipoles are seen as depicted in fig. 1.b. Using the law of force for the dipoles of fig. 1.b, one finds that they attract each other with a force (22). This outcome is an example of the consistency of the laws of physics as seen in the two coordinate frames $\Sigma$ and $\Sigma^{\prime}$.

Let us return to the frame $\Sigma$ and examine two axial magnetic dipoles made of current loops (see fig. 2.a). Each of these dipoles replaces one of the 
dipoles of fig. 1.a and the strength of these dipoles satisfies $M=p$. It turns out that the force exerted on each axial dipole is the same as the force found for the polar dipoles of the previous example (see [7], p. 185). It follows that, like in the case of the polar dipoles depicted in fig. 1.a, the two axial dipoles of fig. 1.b attract each other.

In the second frame $\Sigma^{\prime}$, the current loops are seen as depicted in fig. 2.b. Using the law of force, it is easily seen that in $\Sigma^{\prime}$, like in $\Sigma$, the two dipoles attract each other. Thus, the outcome of the analysis of the two axial dipoles is another example of the consistency of physics.

Let us turn to a hybrid system made of one polar magnetic dipole and one axial magnetic dipole. In the frame $\Sigma$ these dipoles are seen as in fig. 3.a. In this case we examine the interaction of monopoles with velocity fields of charges and vice versa. Let us test the assumption stating:

C. Monopoles interact with velocity fields of charges in the same way as they do with velocity fields of monopoles.

Hereafter, this assumption is called assumption C.

Using assumption $\mathrm{C}$, one finds that the polar dipole, which is made of two displaced monopoles $\pm g$, is seen in $\Sigma$ at $\boldsymbol{r}_{1}=(0,0, r)$. This dipole is pooled downwards, like its counterpart of fig. 1.a. Requiring momentum conservation, one finds that the axial dipole seen in fig. 3.a at $\boldsymbol{r}_{2}=(0,0,-r)$ should be pooled upwards, like its counterpart of fig. 2.a. Thus, it is seen that in $\Sigma$ the two dipoles of fig. 3.a attract each other.

Let us turn to the frame $\Sigma^{\prime}$. In this frame the polar dipole is seen in $\boldsymbol{r}_{1}^{\prime}=(0,0,-r)$. Using the tensorial relations (8)-(11), one finds that its dipole moment points downwards. This is the same situation as seen in fig. 1.b. The axial dipole, which is made of a current loop, is seen in $\Sigma^{\prime}$ at $\boldsymbol{r}_{2}^{\prime}=(0,0, r)$ and its dipole moment points upwards. Thus, it is shown that 
in $\Sigma^{\prime}$, the directions of the dipoles are anti-parallel whereas they are parallel in $\Sigma$. Using assumption $\mathrm{C}$, one finds that, in $\Sigma^{\prime}$, the two dipoles should repel each other. This result contradicts the findings of the right handed frame $\Sigma$.

The contradiction obtained above disproves assumption C. The problem can be settled if one concludes:

D. Monopoles do not interact with velocity fields of charges and charges do not interact with velocity fields of monopoles. Charges and monopoles interact with free waves of both systems.

Conclusion D is consistent with the results of the experiment described in fig. 3. Indeed, in the situation shown in fig. 3.a, as well as in that of fig. 3.b, one finds that if conclusion $\mathrm{D}$ holds then no force is exerted on the dipoles. Thus, the frame $\Sigma$ and $\Sigma^{\prime}$ yield compatible results.

Conclusion D is consistent with the charge-monopole equations of motion obtained in [4] under assumptions A and B, as presented in the introduction. Thus, the particles, equation of motion are

$$
\frac{d P_{(e)}^{\mu}}{d \tau}=F_{(e, w)}^{\mu \nu} J_{(e) \nu}
$$

and

$$
\frac{d P_{(m)}^{\mu}}{d \tau}=F_{(m, w)}^{\mu \nu} J_{(m) \nu},
$$

Here $F_{(e, w)}^{\mu \nu}$ represents the velocity fields of charges, their acceleration fields and free waves emitted from monopoles. Its entries are like those of (2). $F_{(m, w)}^{\mu \nu}$ is defined analogously. Its entries are like those of (5) and (15).

It is found above that only free waves are common to charges and monopoles. These fields satisfy homogeneous Maxwell equations. Thus, Maxwell equa- 
tions (3), (四), (9) and (10) are not affected.

\section{Discussion}

The equations of motion, namely, the Lorentz force (23) and (24) as well as Maxwell equations (3), (40), (9) and (10) are the same as those obtained in [4] by means of assumption B which states that the theory should be derived from a regular Lagrangian function. Two conclusions can be deduced from this outcome:

I. Requirement B is not essential because the same results are obtained without its utilization.

II. The equations of motion can be derived from a regular Lagrangian function.

Conclusion II is obvious. Indeed, it is shown in [4] that the Lagrangian function (written here in the metric of the present work)

$$
\begin{aligned}
L=-\frac{1}{16 \pi} F_{(e, w)}^{\mu \nu} F_{(e, w) \mu \nu}- & J_{(e)}^{\mu} A_{(e, w) \mu}-\frac{1}{16 \pi} F_{(m, w)}^{\mu \nu} F_{(m, w) \mu \nu} \\
& -J_{(m)}^{\mu} A_{(m, w) \mu}+\frac{1}{16 \pi}\left(F_{(w)}^{\mu \nu} F_{(w) \mu \nu}\right.
\end{aligned}
$$

yields the equations of motion obtained here.

Another result of the analysis carried out in the previous section is that an alternative charge-monopole theory whose equations of motion differ from those derived above, must be incompatible with at least one of the postulates used here. Consider, for example, a theory that does not distinguish between electromagnetic fields of charges and those of monopoles and uses 
the following particles' equations of motion[1]

$$
\frac{d P_{(e)}^{\mu}}{d \tau}=F_{(e, m, w)}^{\mu \nu} J_{(e) \nu}
$$

and

$$
\frac{d P_{(m)}^{\mu}}{d \tau}=F_{(e, m, w)}^{* \mu \nu} J_{(m) \nu}
$$

It turns out that in this theory a pure monopole system does not have the ordinary structure of electrodynamics. Here monopole fields are not defined like in (8) as done in electrodynamics but are postulated to have the same physical meaning as fields of charges and are obtained from the later by means of the duality transformation (5). As a result, this theory cannot use potentials of the form (11) that yield a tensor whose entries are like (15). Moreover, regular potentials of electrodynamics of charges (6) cannot be used[10] for monopole fields because, as is well known, in electrodynamics of charges, where fields are derived from potentials like (6)

$$
\nabla \cdot B=\nabla \cdot(\nabla \times A)=0
$$

This property of regular potentials is inconsistent with

$$
\nabla \cdot \boldsymbol{B}=4 \pi \rho_{(m)}
$$

which is the $\mu=0$ equation of (9).

As is well known, potentials are essential ingredients of the electrodynamic Lagrangian function. It follows that the equations of motion (26) and (27) cannot be derived from a regular Lagrangian. This conclusion holds also in the limit of a system of monopoles which does not include electric charges.

Another aspect of the theory that uses (26) and (27) as the equations of motion of charges and monopoles, respectively, is the assignment of pseudoscalar properties to monopoles (see [7], p. 253). Indeed, if monopoles are 
scalars, as used in (8), then the problem presented in fig. 3 persists. On the other hand, if monopoles are pseudo-scalars then the polar dipole of fig. 3.b, which is made of two displaced monopoles, is not as shown in the figure but reverses its direction. Under these circumstances the dilemma apparently disappears.

Consequences of the assignment of pseudo-scalar properties to monopoles affect the construction of a Lagrangian for the equations of motion (26) and (27). Thus, here $j_{(m)}^{\mu}$ is not a 4-vector. It follows that it cannot be used for constructing a scalar with a 4-potential $A_{(e)}^{\mu}$ of charge which transforms like a 4 -vector.

\section{Concluding Remarks}

The discussion carried out in this work relies on the postulate stating that a classical theory of a system which consists of one kind of charges and whose equations of motion are like those of classical electrodynamics, takes a form which is analogous to this theory. Thus, equations (8)-(11) are elements of the theory. In addition to this, it is assumed that a theory of a combined system which consists of several kinds of charges, reduces to the theory of one kind of charge as formulated in (8)-(11), in an appropriate limiting process where all other kinds of charges are removed to infinity.

It is proved that these assumptions lead to the equations of motion of a charge-monopole system (23), (24) and to the obvious form of Maxwell equations (3), (41), (9) and (10). These equations state that charges do not interact with velocity fields of monopoles and monopoles do not interact with velocity fields of charges. Charges and monopoles interact indirectly by 
means of free electromagnetic waves (namely, real photons).

The same equations of motion have been obtained earlier, using a postulate stating that the theory should be derivable from a regular Lagrangian function[4]. It is shown here that this requirement is not essential and that the same results are obtained without relying on the existence of a regular Lagrangian.

It is also proved here that any alternative theory whose equations of motion differ from (23)-(24) cannot satisfy the postulates used here. The theory whose equations of motion are (26) and (27), where charges and monopoles interact with all kinds of fields, is a particular case. Here one uses monopoles that are pseudo-scalars and the pure monopole part of this theory does not take the ordinary form of electrodynamics (8)-(11). 
References:

[1] P. Goddard and D. I. Olive, Rep. Prog. Phys., 41, 1357 (1978) and references therein.

[2] J. R. Ficenec and V. L. Teplitz in Electromagnetism (D. Teplitz, Editor) (Plenum, New York, 1982) and references therein.

[3] F. Rohrlich, Phys. Rev. 150, 1104 (1966).

[4] E. Comay, Nuovo Cimento, 80B, 159 (1984).

[5] H. J. Lipkin and M. Peshkin, Phys. Lett. B179, 109 (1986).

[6] E. Comay, Phys. Lett. B187, 111 (1987).

[7] J. D. Jackson, Classical Electrodynamics (John Wiley, New York, 1975). p. 28.

[8] L. D. Landau and E. M. Lifshitz, The Classical Theory of Fields (Pergamon, Oxford, 1975). p.61.

[9] W. K. H. Panofsky and M. Phillips, Classical Electricity and Magnetism (Addison Wesley, Reading Mass., 1962). p. 19.

[10] P. A. M. Dirac, Phys. Rev. 74, 817 (1948). 


\section{Figure Captions}

Fig. 1:

(a) Two polar magnetic dipoles are held fixed at $\mathbf{r}_{i}=(0,0, \pm r)$, respectively. The signs of the surface monopoles $\pm g$ indicate the direction of the dipole moment.

(b) The system as seen in the left handed laboratory $\Sigma^{\prime}$.

Fig. 2:

(a) Two axial magnetic dipoles $\mathbf{M}$, made of current carrying loops, are fixed like in fig. 1.

(b) The system as seen in the left handed laboratory $\Sigma^{\prime}$.

Fig. 3:

(a) A polar magnetic dipole and an axial one are fixed as shown.

(b) The system as seen in the left handed laboratory $\Sigma^{\prime}$. 



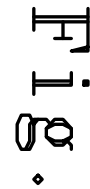

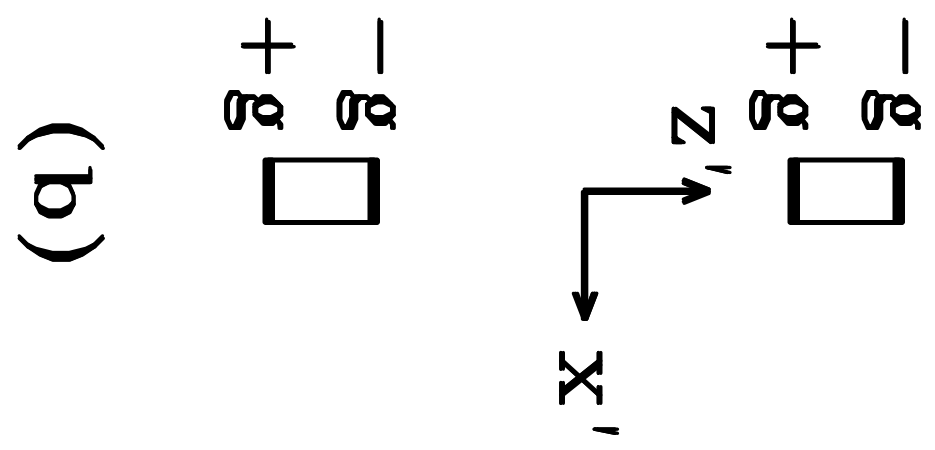





II
oro
.

N

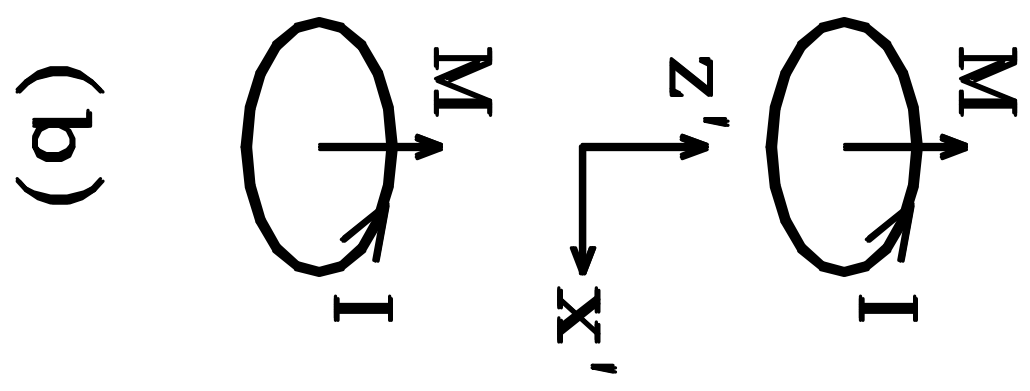






1
$\mapsto$.
00
$\omega$
